\title{
Evaluating dose distributions of normal organs for patients undergoing VMAT therapy of nasopharyngeal carcinoma using Rando phantom and TLD-100H
}

\author{
Yun-Chih Chen ${ }^{\mathrm{a}, \mathrm{b}}$, Hung-Chih Lin ${ }^{\mathrm{b}, \mathrm{c}}$, Wei-Hou Lai ${ }^{\mathrm{c}, \mathrm{d}}$, Hung-Chang Hung ${ }^{\mathrm{e}}$, \\ Hsien-Chun Tseng f,g and Chien-Yi Chen ${ }^{\mathrm{c}, \mathrm{f}, *}$ \\ ${ }^{a}$ Department of Radiation Oncology, Tung's Taichung Metroharbor Hospital, Taichung, Taiwan \\ ${ }^{\mathrm{b}}$ Department of Radiation Oncology, Nantou Hospital, Ministry of Health and Welfare, Nantou, Taiwan \\ ${ }^{\mathrm{c}}$ Department of Medical Imaging and Radiological Sciences, Chung Shan Medical University, Taichung, \\ Taiwan \\ ${ }^{\mathrm{d}}$ Department of Radiology, Dajia Li Hospital, Taichung, Taiwan \\ ${ }^{\mathrm{e}}$ Department of Internal Medicine, Nantou Hospital, Ministry of Health and Welfare, Nantou, Taiwan \\ ${ }^{\mathrm{f}}$ Department of Radiation Oncology, Chung Shan Medical University Hospital, Chung Shan Medical \\ University, Taichung, Taiwan \\ ${ }^{\mathrm{g}}$ School of Medicine, Chung Shan Medical University, Taichung, Taiwan
}

\begin{abstract}
.
BACKGROUND: The routine radiation therapy treatment planning does not include secondary radiation and peripheral doses resulting from radiotherapy exposure in patients with nasopharyngeal carcinoma (NPC) undergoing Volumetric Modulated Arc Therapy (VMAT) using an linear accelerator (linac) of Axesse (Elekta 2538).

OBJECTIVE: VMAT has a better dose conformity of the tumor and is also operated by adjusting the shapes of mulileaf collimator. However, such treatment is potentially important to improve the accuracy of estimated health risks.

METHODS: This study aimed to evaluate the equivalent dose of organ or tissue $\left(D_{T}\right)$ and effective dose $(E)$ for normal organs using the Alderson Rando phantom as an equivalent of the human body. Thermoluminescent dosimeters (TLD-100H) were calibrated by $6 \mathrm{MV}$ X-ray originated by the linac. A total of 252 TLDs were used. These TLDs were inserted into phantom organ or tissue which closely approximated to these places.

RESULTS: The thyroid dose $\left(D_{t h y}\right)$ had the highest dose, $1840 \pm 202 \mathrm{mSv} /$ treatment. The $E$ of the Rando was $7.11 \pm$ $0.61 \mathrm{mSv} /$ treatment, as estimated using ICRP 103 . The skin doses $\left(D_{\text {skin }}\right)$ varied significantly outside the treatment field and decreased as the distance from the treatment field increased.

CONCLUSIONS: This study can be referred to practical guidance regarding radiation protections of the public.
\end{abstract}

Keywords: Volumetric Modulated Arc Therapy (VMAT), nasopharyngeal carcinoma (NPC), Axesse linac, effective dose (E), TLD-100H; Rando, ICRP 103

\footnotetext{
${ }^{*}$ Corresponding author: Chien-Yi Chen, Department of Medical Imaging and Radiological Sciences, Chung Shan Medical University, Taichung 40201, Taiwan. E-mail: chien.ccy@ gmail.com.
}

0928-7329 (c) 2022 - The authors. Published by IOS Press. This is an Open Access article distributed under the terms of the Creative Commons Attribution-NonCommercial License (CC BY-NC 4.0). 


\section{Introduction}

Reported from the statistics of the Ministry of Health and Welfare for 2019 [1], nasopharyngeal carcinoma (NPC) is the $12^{\text {th }}$ leading cause of death among men at Taiwan. NPC is also a head and neck $(\mathrm{H} \& \mathrm{~N})$ cancer among Southeast Asia [1]. The volumetric modulated arc therapy (VMAT) of an Axesse (Elekta 2538) linear accelerator (linac) is a novel effective treatment for many cancers [2,3]. However, the Axesse generates high-energy up to $6 \mathrm{MV}$ radiations during NPC treatment [4-8]. Patients may be exposed to undesirable high energy x-ray primarily due to out-of-field radiations. However, the peripheral dose (PD) is an important issue [4]. Patients always ask physicians as well as radiologist can tell how much extra radiations exposured to normal tissues or organs. To estimate the equivalent doses belong to organ or tissues $\left(D_{T}\right)$ and effective dose $(E)$ are necessary for evaluating the accompanying PD. In this study, Alderson Rando phantom was made by Radiology Support Devices (Long Beach, British Columbia, Canada). The phantom was used as patient surrogates to evaluate $D_{T}$ and $E$ of varying anatomical areas. In order to reduce experimental errors. Quantitatively measured $E$ and $D_{T}$ of patients can provide data for revising plans for treating NPC and determining radiation safety [2-4,9]. Thermoluminescent dosimers (TLD-100H, $3.0 \times 3.0 \times 1.0 \mathrm{~mm}^{3}$ ) were made by Harshaw (Solon, OH, USA). TLDs were inserted into these area.

\section{Method}

\subsection{Volumetric Modulated Arc Therapy (VMAT) via an Axesse linear accelerator (linac)}

For oncology therapy, many different linear accelerator (linac) are used worldwide. The VMAT was designed by Axesse linac of Chung Shan Medical University Hospital (CSMUH). VMAT can associate with better dose conformity to the tumor, superior dose distribution, fewer monitoring units, and faster delivery time $[2,3,5]$. It employs linac to conduct dynamic modulated rotation of intensity-modulated radiation therapy (IMRT). It can operate by adjusting the shapes of multileaf collimator, dose rate, and gantry rotation speed to benefit patients [1].

The Rando weighs $73.5 \mathrm{~kg}$ and is $175 \mathrm{~cm}$ tall. The Rando comprised 35 numbered sections. These could make phantom suitable for oncology dose calculations $[7,10]$. The Rando was embedded in the anthropomorphic material. The densities $\left(\mathrm{g} \mathrm{cm}^{-3}\right)$ of the components were 0.32 for the lungs, 0.98 for the soft tissue, and 2.70 for the skeleton $[3,4,6,10]$.

\section{2. $C T$ simulation and treatment plan}

All of VMAT simulations for CT examination of NPC of phantom were carried by a 16-slice CT sim. The CT-based simulation was carried with the Rando lying supine with a thermoplastic shell. The Philip Pinnacle Planning System 9.0 was adapted for VMAT planning $[3,10]$.

An NPC tumor size of the nasopharyngeal (NP) area of the Rando, $3 \times 3 \times 3 \mathrm{~cm}^{3}$, was simulated at a depth of $1.2 \mathrm{~cm}$ in a central location. In addition, skin marks were tapped in three directions during experiments. Rando was without changing the position during irradiated [6]. Figure 1 displayed the gross tumor volume (GTV) (orange) and the planned target volume (PTV) (red) for the Pinnacle Planning System of CSMUH as well as isodose distributions in the axial scan. Then, the CT organ contours and images were transferred to the Axesse linac. That is $100 \%$ in red and $90 \%$ in green of $200 \mathrm{cGy} /$ treatment of complete prescribed photo doses. All the results were times 28 to $56 \mathrm{~Gy}$ of total NPC treatment. The 


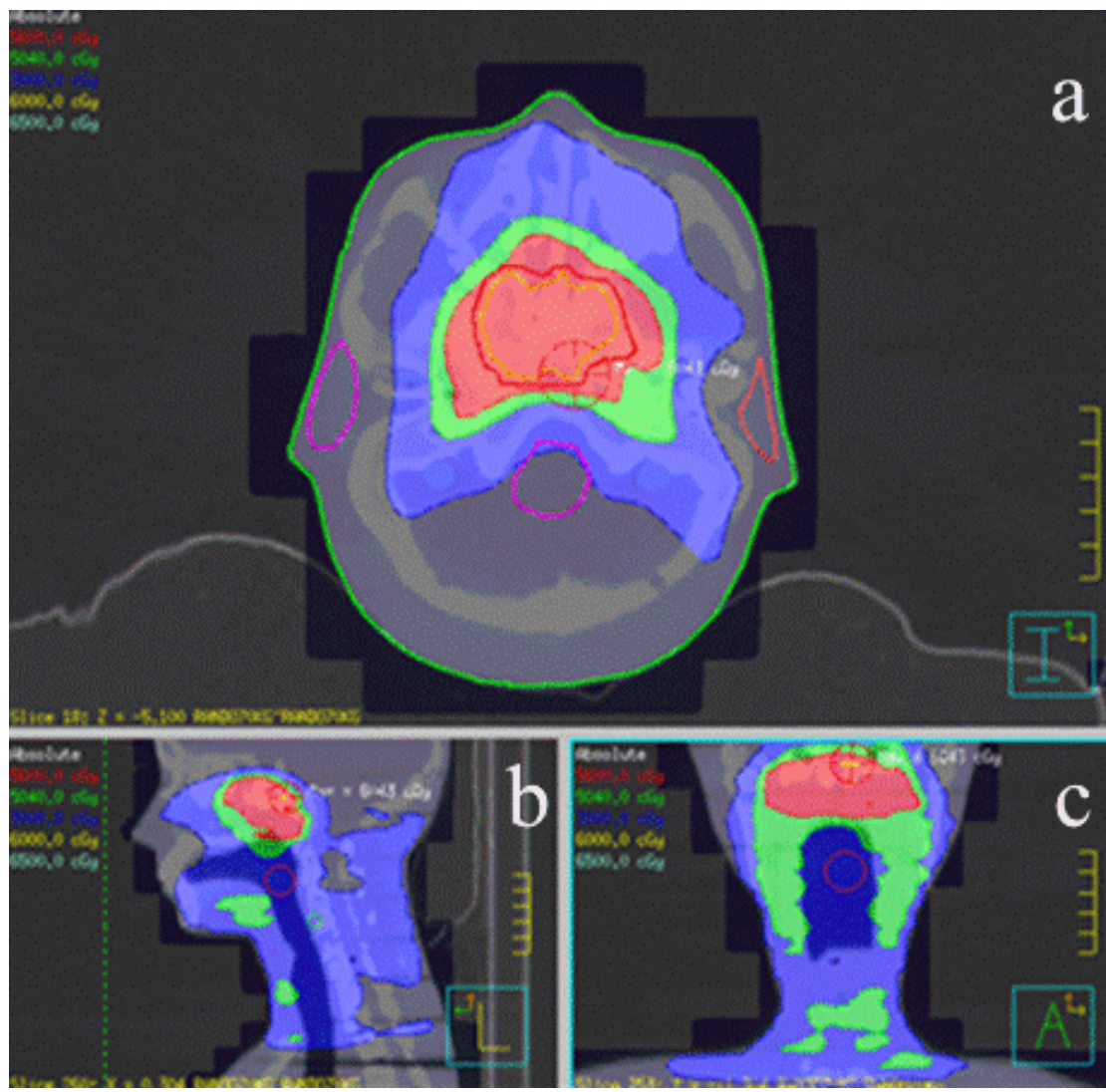

Fig. 1. The NPC treatment of for VMAT (a) axial, (b) sagittal, and (c) coronal of $5^{\text {th }}$ section of Rando views of Pinnacle planning system.

organs at risk (OAR) of Rando included the brainstem and right parotid gland (cobalt violet), left parotid gland and throat (carmine rose), optic nerve (green and brown), spinal cord (light water blue), and lens (gray and blue) [2].

\subsection{Assessment of $E$ and $D_{T}$ values using the thermoluminescent dosimeter approach}

A senior radiologist (H. C. Lin) who had a least 10 years of experience conducted the routine NPC treatment. Only the skin, salivary gland, and brain were directly irradiated during NPC treatment.

The absorbed doses $(D)$ of organs were evaluated at several locations at a fixed distance away from the tumor center, and these data measured from a particular tissue or organ were averaged to represent a representative dose for such organ. In addition, TLDs were inserted into the lens as well as thyroid to evaluate $D_{\text {lens }}$ and $D_{\text {Thy }}$. TLDs were measured in each section of a large organ in plastic bags Such as the brain, and the averaged estimated absorbed dose was taken as $D_{\text {brain }}$. Furthermore, for smaller organs, likes the lens, absorbed doses were obtained by averaging 3 TLDs in 1 bag [9].

For calculation of $D_{T}$ and $E$ for a large tissues or organs, the following equation is used to determine $D_{T}$ which was recommended by ICRP 103 .

$$
D_{T}=D \times W_{R}
$$


Table 1

Measurement locations of the 52 points, the weighting factors $\left(W_{T}\right)$ of organs or tissues are recommended by ICRP 103 (Eqs (1) and (2)

\begin{tabular}{lllr}
\hline Section of rando & Organ/tissue & $W_{T}$ & Numbers of TLD \\
\hline $1 \sim 32$ & Skin & 0.01 & 96 \\
$1 \sim 5$ & Brain & 0.01 & 15 \\
3 & Lens & 0.017 & 6 \\
$4,5,24,30$ & Bone surface $^{*}$ & 0.01 & 39 \\
6 & Oral mucosa* & 0.017 & 3 \\
6,7 & Salivary glands & 0.01 & 12 \\
8,31 & Lymph nodes* & 0.017 & 3 \\
$8,25,26,27$ & Bone marrow & 0.12 & 12 \\
9 & Esophagus & 0.04 & 6 \\
10 & Thyroid & 0.04 & 6 \\
$11 \sim 15,17$ & Lungs & 0.12 & 21 \\
16 & Heart* & 0.017 & 3 \\
18 & Breast & 0.12 & 3 \\
18 & Stomach/Spleen & 0.12 & 3 \\
19 & Pancreas* & 0.017 & 3 \\
20 & Liver & 0.04 & 3 \\
21,29 & Colon & 0.12 & 6 \\
22 & Kidney* & 0.017 & 3 \\
23 & Small intestine* & 0.017 & 3 \\
28 & Bladder & 0.04 & 3 \\
32 & Gonads & 0.08 & 3 \\
& Total & 1.000 & 252 \\
\hline
\end{tabular}

${ }^{*}$ Remainder including: Lens, Oral mucosa, Lymph nodes, Heart, Pancreas, Kidney, Small intestine.

$D$ is absorbed doses, $W_{R}=1 \mathrm{~Sv} / \mathrm{Gy}$, for X-rays coming from linac. WR is the radiation weighting factor. In order to evaluate the effective dose, $E, D_{T}$ and $W_{T}$ are as following:

$$
E=\Sigma D_{T} \times W_{T}
$$

Table 1 lists explicitly some organs and tissues. In addition, others are "remainder tissues". To make doses evaluations for these tissues or organs those were near to as well as within the radiation field. From the head to the gonads, including the remainder organs, could be used to evaluate $E$. A total of 252 TLDs were used. 3 TLDs were inserted into each bag to minimize the errors, and to obtain the $D_{T}$ at a particular location/organ. The $D_{T}$ were evaluated by inserting TLDs into the Rando at locations due to different organs $[9,10]$.

In addition, 42 TLDs were inserted into the $\mathrm{H} \& \mathrm{~N}$ of the patients. The background radiation was measured by 9 TLDs. Finally, total errors could be effectively suppressed by conducting 3 independent trials. This is 32 bags of TLDs attached to the Rando' skin. The measured points on these organs were recommended by ICRP 103. The corresponding values of $W_{T}$ was listed in Table 1 [2,5].

\subsection{Calibrate TLDs via $6 \mathrm{MV}$ beams}

These TLDs of photon dose measurement and linearity were calibrated via Axesse linac (6 MV). (1) The distance from source-to-surface is $100 \mathrm{~cm}$. (2) At a field size of $10 \times 10 \mathrm{~cm}^{2}$, depth of $1.5 \mathrm{~cm}$ in solid water were used. To lower the experimental uncertainty, solid water (CIRS, Norfolk, VA, USA) was used rather than water. Calibrated TLDs were exposured at doses ranging from 10 to $400 \mathrm{cGy}$ that included the prescribed NPC fraction dose, $200 \mathrm{cGy} /$ treatment herein. An ionization chamber (IC), type 
Table 2

Random and systematic errors coming from the practical evaluation of radiation

\begin{tabular}{lc}
\hline Source & One standard deviation $\Delta_{i}$ \\
\hline Measurement: & \\
TLD counting statistics & $3 \% \sim 10 \%$ \\
Others: & \\
$W_{T}$ (weighting factor) & $5 \%$ \\
VMAT output photon dose & $<2 \%$ \\
TLD-100H calibration & $3 \% \sim 20 \%$ \\
Inserting positions of phantom organ or tissue & $5 \%$ \\
Density effects of PMMA phantom & $1.5 \% \sim 3.8 \%$ \\
Variation of Harshaw 3500 reader & $3 \% \sim 8 \%$ \\
$\Delta_{t o t}$ & $9.00 \% \sim 21.0 \%$ \\
\hline
\end{tabular}

NE 2571 (Nuclear Enterprises, UK). It was positioned in the solid water as published elsewhere [3,4]. The IC had a volume of $0.6 \mathrm{~mL}$.

The TLDs were used because of their small dimensions, and the lower dependence of its response on dose rate, photon energy, as well as the direction of any incidence of X-rays from Axesse linac. A calibrated TLD readout system of Harshaw 3500 reader could be useful to measure extra X-rays radiations, and evaluate specific doses with better reliability. After 1 day of exposure, these irradiated TLDs were measured by an automated Harshaw 3500 reader. The readout was (1) the TLD was heated up to $50^{\circ} \mathrm{C}$, then hold $1 \mathrm{sec}$. (2) Heated up to $240^{\circ} \mathrm{C}$ under a rate of $10^{\circ} \mathrm{C} / \mathrm{s}$ by applying $780 \mathrm{~V}[3,4,9,10]$. In addition, after annealing process, these TLDs could be reused [2-4,9].

\subsection{In vivo experiment undergoing NPC treatment}

OAR in and nearby the NP where the primary irradiated were mainly measured, then throughout the entire body. Totally, 252 TLDs were placed in the Rando at each section, then directly used to evaluate $D_{T}$ in sections from 4 to 9 of the $H \& N$. For all measured points, the final $D_{T}$ value was evaluated by mean 3 TLDs in each bag [9]. The $D_{T}$ of the Rando were mean to evaluate a representative response of that organ, and the averaged $D_{T}$ at the same distance from the central axis for each organ was used (refer to Table 1). For large organs, like the brain and the lung, TLDs were inserted into each section, the averaged dose was $D_{\text {breast }}$ and $D_{\text {lung }}$, respectively. For small organs, such as the gonad, the averaged dose was named as $D_{\text {gonad }}$. The error bars included the uncertainty of these $D_{T}$ across different TLD-100H in these organ or tissue.

\section{Results and discussion}

The NPC treatments exposure several organs via many radiation sensitivities. The $E$ considered how much extra radiations were calculated from an individual tissue and the relative sensitivity of the tissue. The $E$ of NPC treatments were evaluated via the TLD-100H method.

\subsection{Calibration and uncertainty of TLD-10OH}

The TLD-100H represented linearly of radiation doses ranging 10-400 cGy.

The conversion factor of the TLD-100H was $\mathrm{Y}(\mathrm{cGy})=1.2876 \times \mathrm{TLD}(\mu \mathrm{C})+5.1925$ obtained via the EXCEL. Additionally, $R^{2}$ was 0.9810 [3].

These accuracy and precision of the $D_{T}$ measurements via TLD-100H method are evaluated by many factors. Table 2 lists the systematic and random errors. 


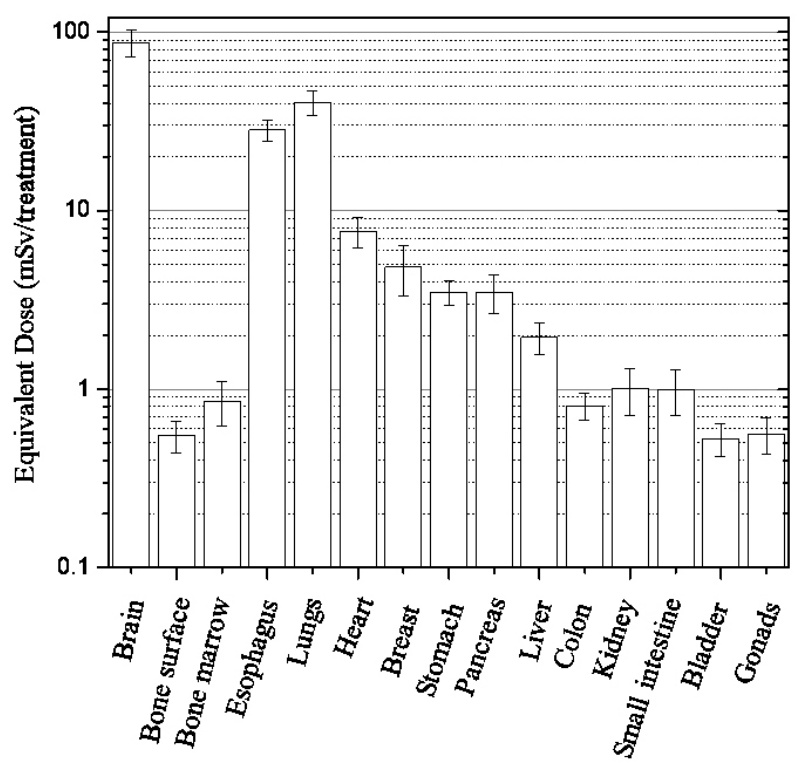

Fig. 2. Equivalent doses (mSv/treatment) of organs of Rando during NPC treatment. $D_{T}$ was evaluated by inserting many TLDs into each organ. Mean values and errors shown as bars.

\subsection{The equivalent doses $\left(D_{T}\right)$ and effective doses $(E)$ of normal organs or tissues}

This study measured the $D_{t h y}$ value of the thyroid at section $10^{\text {th }}$ of the phantom. The $D_{t h y}$ value had the highest dose compared with other organs, that is, $1840 \pm 202 \mathrm{mSv} /$ treatment. In fact, $D_{\text {thy }}$ of thyroid, a small difference in the positions because of one or a few TLDs nearly to the treatment region. Figure 2 shown the measured $D_{T}$ of the Rando. The $D_{T}$ were high for lung, esophagus, and heart during the treatment.

Parts of the oral mucosa, lymph nodes, and skin were also scanned. In addition, these organs had relatively low $W_{T}$ (Table 1 ). The $E$ and $D_{T}$ were calculated recommended by ICRP 103 [5,11].

The $E$ for NPC treatment was large, up to $7.11 \pm 0.61 \mathrm{mSv} /$ treatment. Both $E$ and $D_{T}$ values obtained were in agreement with those in previous studies [6].

\subsection{Skin dose $\left(D_{\text {skin }}\right)$}

Figure 3 plots 32 measurements, data reflected the skin dose $\left(D_{\text {skin }}\right)$ or entrance surface air kerma at any point in the Rando. The $D_{\text {skin }}$ of NPC treatment were evaluated via 96 TLDs those were attached to the skin. Figure 3 plots $D_{\text {skin }}$ as a function of results over 3 trials, based on the sections from the treatment edge of the Rando. Due to extra irradiation, the $D_{\text {skin }}$ at the periphery of the treatment volume had huge errors. The $D_{\text {skin }}$ value ranged from $26.9 \pm 3.24\left(1^{\text {st }}\right.$ section $)$ up to $1340 \pm 200\left(12^{\text {th }}\right)$, then down to $0.82 \pm 0.29$ (32 $\left.2^{\text {nd }}\right) \mathrm{mSv} /$ treatment. Such as (1) lens $57.8 \pm 8.2 \mathrm{mSv} /$ treatment $\left(2^{\text {nd }}\right)$; (2) thyroid $687 \pm 61 \mathrm{mSv} /$ treatment $\left(13^{\text {th }}\right)$; (3) lung $50.3 \pm 7.6 \mathrm{mSv} /$ treatment $\left(15^{\text {th }}\right)$; and (4) gonad $0.90 \pm 0.17$ $\left(31^{\text {th }}\right) \mathrm{mSv} /$ treatment. The out-of-field doses, that is skin doses, displayed a gradual reduction in the $D_{\text {skin }}$ with increasing distance from the treatment edge during NPC treatment. Finally, the $D_{\text {skin }}$ depended on whether the skin was in or out of the X-rays directly or not. According to the NPC treatment, an organ or tissue not close to the treatment field received approximately the background radiation. The radiation doses were significant near the treatment edge. Those would be became negligible due to the distances far from $47 \mathrm{~cm}$ (Fig. 3). 


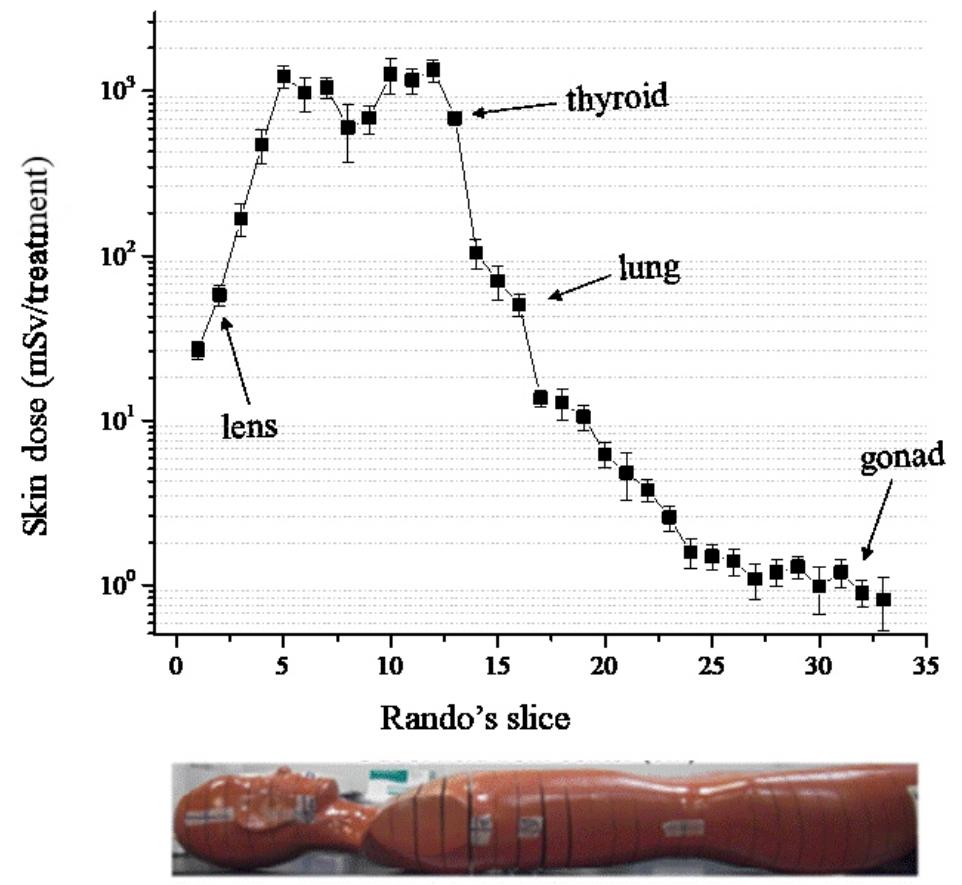

Fig. 3. Assessing the skin dose $\left(D_{\text {skin }}\right)$ vs. Rando's section during NPC treatment.

\subsection{Comparison of results with those of other studies}

Notably, the $D_{T}$ value was higher than that for other organs/tissues near NPC. $D_{T}$ were decreased from the treatment field increased. D'Agostino et al. reported an intercomparison designed to PD during H\&N treatment. The $D_{T}$ values were compared with that obtained through VMAT-6 MV (Varian clinic 2100 CD) and TT-6MV of Tomotherapy for NPC treatment [3]. Mutic and Low evaluated the whole-body (WB) dose via the TLD method and water phantom. The WB dose was $315 \mathrm{mSv} / 70 \mathrm{~Gy}$ at $40 \mathrm{~cm}$ from the target's edge of H\&N cancer. This was nearly equal to $0.043 \%$ tumor center dose [12]. Betta et al. evaluated $2.3 \mathrm{mSv}$ (stetrnumaid), and $1.7 \mathrm{mSv}$ (upper parts of lung) during 5 Gy of stereotactic treatment using the Rando [13].

\subsection{Limitations}

This study had many limitations. (1) Lack of female breast phantom could be used, so the $D_{\text {breast }}$ values were calibrated; one bag (three TLDs) was attached $1 \mathrm{~cm}$ under the skin surface of the $11^{\text {th }}$ section of Rando. (2) These organ doses evaluated at the thyroid and skin might not accurately reflect that delivered to these organs, as parts of TLDs might have been irradiated. Many uncertainties were seen in one bag. Other TLDs had low count rates. Furthermore, the TLDs were distributed inserted into organs/tissue and could be uniformly. These TLDs were positioned at the same locations during experiments.

\section{Conclusions}

This study evaluated the $E$ and $D_{T}$ values during NPC treatment using Rando undergoing VMAT of Axesse linac. The $D_{T}$ of the lung, esophagus, and heart were very high, and different significance from 
those of other organs. $D_{\text {thy }}$ were high, up to $1840 \pm 202 \mathrm{mSv} /$ treatment. The $D_{\text {skin }}$ for the periphery of the treatment volume had significant variations due to extra irradiations. It was ranging from $26.9 \pm 3.24$ $\left(1^{\text {st }}\right)$ up to $1340 \pm 200\left(12^{\text {th }}\right)$, and then down to $0.82 \pm 0.29\left(32^{\text {nd }}\right) \mathrm{mSv} /$ treatment.

\section{Acknowledgments}

The authors want to thank the staff of Chung Shan Medical University Hospital for their efficient support.

\section{Conflict of interest}

None to report.

\section{References}

[1] Statistics of General Health Welfare of 2019, Ministry of Health and Welfare, 2019. (https://dep.mohw.gov.tw/DOS/cp1735-3242-113.html).

[2] Lai WH. Evaluating Dose Distributions and Minimum Detectable Dose of TLD-100H Approach for Nasopharyngeal Carcinoma Based on Rando and SDM Phantoms undergoing VMAT. Ms Thesis, Chung Shan Medical University Taiwan. 2015.

[3] D'Agostino E, Bogaerts R, Defraene G, et al. Peripheral doses in radiotherapy: A comparison between IMRT, VMAT and Tomotherapy. Radiat Meas. 2013; 57: 62-67.

[4] Lin HC, Lai TJ, Tseng HC, et al. Radiation doses with various body weights of phantom in brain 128-slice MDCT Examination. J Radiat Res. 2019; 60(4): 466-475.

[5] International Commission on Radiological Protection, Recommendations of the International Commission on Radiological Protection. Publication 103, Annals of ICRP Vol. 37 (2/4), Pergamon Press, Oxford; 2007.

[6] Lee FKH, Yip CWY, Cheung FCH, Leung AKC. Dosimetric difference amongst 3 techniques: TomoTherapy, slidingwindow intensity-modulated radiotherapy (IMRT), and RapidArc radiotherapy in the treatment of late-stage nasopharyngeal carcinoma (NPC). Med Dosim. 2014; 39: 44-49.

[7] Lee TF, Ting HM, Chao PJ, Fang FM. Dual arc volumetric-modulated arc radiotherapy (VMAT) of nasopharyngeal carcinomas: a simultaneous integrated boost treatment plan comparison with intensity-modulated radiotherapies and single arc VMAT. Clin Oncol. 2012; 24: 196-207.

[8] Lu SH, Cheng JCH, Kuo SH, Lee JJS, Volumetric modulated arc therapy for nasopharyngeal carcinoma: a dosimetric comparison with TomoTherapy and step-and-shoot IMRT. Radiother Oncol. 2012; 104: 324-330.

[9] Tseng HC, Liu WS, Tsai HH, et al. Radiation dose for normal organs by helical tomotherapy for lung cancer. Appl Radiat Isot. 2015; 102: 35-41.

[10] Chen LF, Tseng HC, Pan LK, et al. Evaluating environment radiations at Axesse linac undergoing NPC treatment of VMAT. Comput. Aided Surg. 2016; 21(S1): 79-83.

[11] Chung Shan Medical University, Available from: http://english.csmu.edu.tw/bin/home.php. 2021.

[12] Mutic S, Low D. Whole-body dose from tomotherapy delivery. Int J Radiat Oncol Biol Phys. 1998; 42: 229-232.

[13] Betta DE, Fariselli L, Bergantin A, et al. Evaluation of the peripheral dose in stereotactic radiotherapy and radiosurgery treatments. Med Phys. 2010; 37: 3587-3594. 